\title{
Respostas de Agressividade no Rorschach (R-PAS) de Homens Autores de Violência Conjugal
}

\author{
Sonia Liane Reichert Rovinski ${ }^{1}$, Andréia Mello de Almeida Schneider \\ Universidade Federal do Rio Grande do Sul, Porto Alegre-RS, Brasil \\ Juliane Pariz \\ Griffith University - Gold Coast, Queensland, Australia \\ Álvaro Zaneti Santos, Denise Ruschel Bandeira \\ Universidade Federal do Rio Grande do Sul, Porto Alegre-RS, Brasil
}

\section{RESUMO}

Este artigo analisa a validade dos indicadores de agressividade do Rorschach em uma amostra forense. São participantes deste estudo 31 homens agressores violentos contra mulheres, os quais foram indiciados, julgados no Tribunal de Violência Doméstica e Familiar e, posteriormente, encaminhados a um Grupo Reflexivo de Gênero. O Rorschach foi administrado por meio do sistema R-PAS, de acordo com a padronização e normas propostas pelo manual, com excelente confiabilidade entre avaliadores para os dois códigos analisados. Os índices encontrados para AGC e AGM foram comparados aos dados normativos por meio do teste $t$ de Student. Apenas AGC mostrou diferença significativa em relação à amostra normativa, com menores resultados. O AGM não evidenciou validade para diferenciar os grupos.

Palavras-chave: teste de Rorschach; psicologia forense; violência doméstica.

\section{ABSTRACT - Rorschach Aggression Responses in Male Perpetrators of Conjugal Violence}

This article analyzes the validity of Rorschach's Aggression indicators in a forensic sample. Study participants are thirty-one men who were violent aggressors against women, who were indicted, tried in the Domestic and Family Violence Court, and later referred to a Reflexive Gender Group. Rorschach was administered through the Rorschach Performance Assessment System (R-PAS), according to the standardization and norms proposed by the manual, with excellent inter-rater reliability for the two codes analyzed. The indexes found for AGC and AGM were compared to the normative data by the Student's t-test. Only AGC showed a significant difference in relation to the normative sample, with lower results. The AGM did not show validity to differentiate the groups.

Keywords: Rorschach test; forensic psychology; domestic violence.

\section{RESUMEN - Respuestas de Agresividad de Rorschach (R-PAS) de Hombres Autores de Violencia Conyugal}

Este artículo analiza la validez de los indicadores de agresividad de Rorschach en una muestra forense. Participaron de este estudio 31 hombres agresores violentos contra mujeres, que fueron acusados, juzgados en el Tribunal de Violencia Doméstica y Familiar y luego encaminados a un Grupo Reflexivo de Género. El Rorschach se administró a través del sistema R-PAS, de acuerdo con la estandarización y las normas propuestas por el manual, con excelente confiabilida dentre evaluadores para los dos códigos analizados. Los índices encontrados para AGC y AGM se compararon con los datos normativos por medio del test t- de Student. Solo AGC mostró una diferencia significativa en relación con la muestra normativa, con menores resultados. El AGM no evidenció validez para diferenciar los grupos.

Palabras clave: test de Rorschach; psicología forense; violencia doméstica.

O processo de avaliação psicológica tem por objetivo fornecer informações para orientar a tomada de decisões sobre o indivíduo que está sendo avaliado. Nessas situações, é comum que os profissionais se utilizem de testes psicológicos a fim de explorar diversas características a respeito do modo de pensar, sentir e agir do sujeito. Quando a utilização de testes se dá no contexto forense, deve-se considerar a influência dos fatores coercitivos na validação de seus resultados, pois o avaliado, com receio das consequências do processo, pode vir a interferir, de forma intencional ou não, na produção de suas respostas (Rovinski, 2013; Nørbech, Fodstad, Kuisma, Lunde, \& Hartmann, 2016).

Dentre os instrumentos de avaliação da personalidade mais pesquisados no contexto forense, encontra-se o Rorschach que, além de oferecer medidas válidas 
e confiáveis, possibilita a observação da personalidade em ação (Benjestorf, Viglione, Lamb, \& Giromini, 2013; Erard, 2012; Erard, Meyer, \& Viglione, 2014; Meyer, Viglione, Mihura, Erard, \& Erdberg, 2017; Society for Personality Assessment, 2005; Viglione \& Meyer, 2008). A versão mais atual do teste, denominada R-PAS (Sistema de Avaliação por Performance no Rorschach), oferece uma descrição do avaliado que é baseada em sólidas evidências de qualidade do instrumento (Mihura, Meyer, Bombel, \& Dumitrascu, 2015; Mihura, Meyer, Dumitrascu, \& Bombel, 2013, 2016). Diferentemente dos instrumentos de autorrelato, o Rorschach contorna a limitação quanto ao que a pessoa é capaz, ou ao que ela está disposta a dizer sobre si mesma, ou o quão consciente ela está de suas próprias características (Erard \& Evans, 2017; Meyer et al., 2017). Além disso, os estímulos oferecidos pelos cartões do Rorschach possibilitam que a análise do desempenho do avaliado, ao resolver o problema proposto pela tarefa de responder ao que a mancha de tinta se parece, envolve uma perspectiva tanto nomotética quanto idiográfica (Meyer et al., 2017).

Todas essas características, ainda somadas ao fato de que o R-PAS, podem ser interpretadas de acordo com diferentes teorias psicológicas, fazem, portanto, desse teste um instrumento de extrema relevância ao contexto jurídico, pois satisfaz as necessidades e os questionamentos mais comuns da justiça quanto à análise do comportamento baseada em evidências (Erard \& Evans, 2017; Gacono, Evans, \& Viglione, 2008; Weiner, 2001; Rovinski, 2013). Dentre os temas de interesse dos profissionais que trabalham junto aos tribunais, e que já vem sendo objeto de estudo em pesquisas internacionais, encontra-se o da validade dos indicadores de agressividade, principalmente quanto à possibilidade de estarem associados a condutas de violência no contexto do mundo real.

O R-PAS surgiu a partir do Sistema Compreensivo (SC), proposto por Exner, mas também inclui outros estudos empíricos que fundamentam os indicadores de agressividade (AGC e AGM) agora propostos (Meyer et al., 2017). No SC, há apenas um indicador específico de agressividade, o Código Especial AG, que é codificado para respostas que envolvem movimento agressivo. Contudo, Gacono e Meloy (1994), desde a década de 1990, já mostravam a necessidade de ampliar esse tipo de avaliação por meio da pontuação de outros indicadores: conteúdos agressivos (AGC), agressividade potencial (AGPot), agressividade passada (AGPast) e sadomasoquismo (SM). Kivisto e Swan, (2013) estudaram esses indicadores e apontaram uma associação positiva com comportamento agressivo $(r=0,40, p=0,02)$ e Katko, Meyer, Mihura e Bombel (2010) sugerem o uso do AGC como preditor de agressividade. Assim, na atual proposição do R-PAS, além de manter-se a variável AGM, equivalente ao AG do SC, passou-se a incluir na codificação a identificação dos conteúdos agressivos (AGC), antes abreviado por Gacono e Meloy como AGC (Gacono \& Meloy, 1994; Meyer et al., 2017).

Apesar de tanto o AGC quanto o AGM, no R-PAS, estarem dentro do domínio percepção de si e outros, no AGC a agressão está associada a uma característica do objeto e indica preocupações agressivas por parte do sujeito. Já, no AGM, a agressão reside na natureza da ação ou movimento do objeto e aponta para uma possível presença de impulso agressivo (Meyer et al., 2017). Esses códigos parecem se complementar por abordar diferentes aspectos da personalidade, um mais voltado para o pensar e o outro mais voltado para o impulso, a ação.

Como o R-PAS é um sistema recente, ainda não existem artigos empíricos brasileiros conduzidos especificamente no contexto forense que abarquem nomeadamente as evidências de validade dos códigos AGC e AGM. Contudo, pesquisas empíricas realizadas com SC têm sustentado que o código AG, equivalente ao AGM do R-PAS (Meyer et al., 2017), estaria relacionado à intensidade do impulso agressivo, mas poderia oscilar quanto à sua frequência, considerando a tensão gerada pelo caráter egossintônico ou egodistônico do impulso (Gacono \& Meloy, 1994). De acordo com esses autores, o código AGC, o mais frequente do conjunto dos escores ampliados sobre a agressividade, mostrou-se associado a identificação, ideação ou preocupação agressiva, o que atualmente aparece relacionado à interpretação do AGC no R-PAS (Meyer et al., 2017). Em relação à predição de comportamentos agressivos, observou-se que o código AG, do SC, teria poder preditivo negativo, sendo mais efetivo em excluir casos com histórico de comportamentos agressivos, quando comparado ao código AGC proposto por Gacono e Meloy (1994). Este último código, por sua vez, teria poder preditivo positivo, sendo mais efetivo na identificação de agressores (Gacono, Bannatyne-Gacono, Meloy, \& Baity, 2005). Além disso, Gacono et al. (2008) indicam que o AGC sofre menor interferência do contexto coercitivo, sendo mais eficaz na identificação de casos com histórico de violência. Contudo, o Manual do R-PAS salienta a necessidade de se considerar o contexto da avaliação ao interpretar o AGC e o AGM, pois são códigos que os avaliados costumam perceber e podem suprimi-los ou maquiá-los para não se mostrar agressivos (Meyer et al., 2017).

Um estudo conduzido por Benjestorf et al. (2013) com o SC, que analisou as codificações para agressividade propostas por Gacono e Meloy (1994), evidenciou que, em uma condição coercitiva simulada, as respostas AG e AGC seriam mais elevadas em sujeitos agressores, ainda que a diferença entre médias não se apresentasse de forma significativa ( $\mathrm{AG}$ agressores $M=0,76$; $\mathrm{AG}$ não agressores $M=0,30$; AGC agressores $M=2,62$; não agressores $M=2,60)$. No entanto, os participantes produziram aproximadamente metade da quantidade de respostas agressivas quando solicitados a se mostrarem não agressivos, sugerindo uma redução de AGC quando motivado 
a agir desse modo, o que corrobora o alerta do manual do R-PAS quanto à manipulação de respostas. Já o estudo conduzido por Nørbech et al. (2016) evidenciou AG mais elevado no grupo de pessoas sem histórico de violência que no grupo de agressores e AGC sem diferença significativa entre os grupos.

$\mathrm{Na}$ realidade brasileira, essa discussão sobre indicadores de agressividade em instrumentos psicológicos mostra-se bastante incipiente, principalmente quando se buscam evidências que possam auxiliar, no contexto forense, na prevenção de novos incidentes com vítimas vulneráveis. O contexto da violência doméstica carece de investigações que tenham o agressor como foco de pesquisa, evidenciando uma verdadeira lacuna na literatura (Silva, Coelho, \& Moretti-Pires, 2014). Assim, a presente pesquisa buscou evidências de validade de critério da interpretação dos códigos específicos de agressividade propostos pelo R-PAS (AGM e AGC), por meio da comparação dos escores de uma população de homens autores de violência contra a mulher com os dados normativos.
Método

\section{Participantes}

Os participantes constituíram-se em um grupo de 31 homens autores de violência, que foram indiciados e julgados em processos junto ao Juizado de Violência Doméstica e Familiar contra a Mulher. Estes foram encaminhados pelo juiz para participar de um Grupo Reflexivo de Gênero coordenado por uma psicóloga judicial dentro do próprio Foro, onde foram convidados para esta pesquisa. A idade dos participantes situou-se entre 20 e 65 anos $(M=42 ; D P=12,3)$. A escolaridade oscilou entre fundamental incompleto a superior completo, sendo a faixa mais frequente a de ensino médio completo e incompleto ( $42 \%$ ), seguido do ensino fundamental completo e incompleto (35\%). O estado civil predominante foi o de solteiro (48\%), seguido dos casados (32\%). Mais de $80 \%$ dos sujeitos trabalhava, com renda entre 1 a 5 salários mínimos. Nenhum dos sujeitos informou estar respondendo a outros tipos de processo judicial, além daquele de violência contra sua companheira.

Tabela 1

Distribuição em Termos de Frequência e Porcentagem dos Sujeitos da Amostra quanto à Escolaridade ( $n=31)$

\begin{tabular}{lcc}
\multicolumn{1}{c}{ Escolaridade } & $f$ & $\%$ \\
\hline Ensino fundamental incompleto e completo ( $\leq 8$ anos de estudo) & 11 & 35,48 \\
Ensino médio incompleto e completo (de 9 a 11 anos de estudo) & 13 & 41,94 \\
Superior incompleto (de 11 a 15 anos de estudo) & 7 & 22,58 \\
\hline Total & 31 & $100 \%$ \\
\hline
\end{tabular}

\section{Procedimentos e Instrumentos}

Na dinâmica do Juizado de Violência Doméstica e Familiar contra a Mulher, os sujeitos encaminhados ao grupo de reflexão passavam, inicialmente, por uma entrevista com a psicóloga judicial, podendo decidir sobre sua participação neste trabalho. Os grupos funcionavam com um número fixo de 12 encontros. O convite para participação na pesquisa foi feito entre o $4^{\circ}$ e o $8^{\circ}$ encontro, período em que geralmente os sujeitos se encontravam menos resistentes à participação na pesquisa, mas ainda envolvidos com o andamento do grupo. Após o aceite para participação, foi marcado com cada um dos participantes um horário para a aplicação dos instrumentos em período imediatamente anterior ou posterior ao horário do grupo, evitando deslocamentos extras ao Foro. Nessa entrevista, os participantes assinavam o Termo de Consentimento Livre e Esclarecido (TCLE) para, em seguida, responderem ao questionário sobre dados pessoais e à aplicação do Rorschach ${ }^{2}$. O projeto de pesquisa foi aprovado por Comitê de Ética em Pesquisa (Parecer
747.928, em 14/08/2014), atendendo às exigências das Resoluções do Conselho Nacional de Saúde no 196, de 10/10/96 e n. 251, de 07/08/97.

Para a coleta dos dados, participaram três pesquisadoras, todas com treinamento na técnica e integrantes do grupo de estudos sobre o R-PAS em nível de pós-graduação.

Os protocolos do Rorschach foram codificados conjuntamente pelas três pesquisadoras e, após essa etapa, foram sorteados sete casos (22\%) que foram enviados para análise de um juiz para a condição e proficiente para codificação e aplicação do R-PAS. De posse das duas codificações (pesquisadoras e juiz), foi calculado o índice de concordância intraclasse (Intraclass Correlation Coefficient - ICC). Segundo Exner e Sendín (1997), se a concordância entre o psicólogo que aplica o instrumento e o juiz for boa em pelo menos $20 \%$ dos casos da amostra, é possível que o pesquisador faça a codificação dos demais sem a necessidade da avaliação de todos os protocolos por juízes. Foram considerados pobres os valores $<0,40$; moderados de 0,40 a 0,59 ; bons 
de 0,60 a 0,74 e excelentes $>0,75$ (Cicchetti, 1994). As discordâncias entre avaliadores e juiz foram tratadas por meio de uma discussão conjunta para se decidir pela melhor codificação, com base nas diretrizes de do R-PAS (Meyer et al., 2017). Todas as informações coletadas foram organizadas em banco de dados e analisadas a partir de técnicas de estatística descritiva e inferencial. Para tanto, foi utilizado o programa estatístico Statistical Package for the Social Sciences (SPSS).

O levantamento das variáveis dos protocolos do Rorschach foi feito em sua totalidade, utilizando a codificação on-line do R-PAS. No entanto, para a presente pesquisa, foram considerados apenas os indicadores relacionados à categoria de agressividade: AGM (movimento agressivo que envolve intenção ou motivo agressivo frente ao outro) e AGC (conteúdo agressivo, que reflete preocupações e identificações com conteúdos agressivos) (Meyer et al., 2017).

O teste $t$ foi utilizado como objetivo de comparar as médias de AGC e AGM da amostra de homens autores de violência contra a mulher com a média da população normativa do manual do R-PAS, da qual fazem parte protocolos coletados na população brasileira em geral. $\mathrm{O}$ tamanho do efeito foi avaliado pelo $d$ de Cohen, sendo $<0,19$ insignificante; de 0,20 a 0,49 pequeno; de 0,50 a 0,79 médio e de >0,80 grande (Cohen, 1988, p. 40).

\section{Resultados e Discussão}

Os valores de correlação intraclasse (ICC) foram calculados para estabelecer a concordância entre avaliadores. Os resultados obtidos para os conteúdos agressivos AGC e AGM, a partir da análise da concordância entre a avaliação das pesquisadoras e de um juiz, estão indicados na Tabela 2 a seguir.

Tabela 2

Estatística ICC para Conteúdos AGM e AGC do R-PAS

\begin{tabular}{lcc}
\hline \multicolumn{1}{c}{ Variáveis } & ICC & IC (95\%) \\
\hline AGC & 0,84 Excelente & $0,78-0,88$ \\
AGM & 0,89 Excelente & $0,84-0,92$ \\
\hline
\end{tabular}

O grau de confiança entre os avaliadores, tanto para AGC quanto para AGM é considerado excelente. Ambos são superiores aos encontrado em estudos recentes (Kivisalu, Lewey, Shaffer, \& Canfield, 2016; Pignolo et al., 2016). O resultado da comparação de médias entre o grupo de homens autores de violência contra a mulher, em relação à amostra normativa do R-PAS (Meyer et al., 2017, p. 353) e o tamanho do efeito estão descritos na Tabela 3 .

A análise dos dados da tabela demonstra que os homens autores de violência contra a mulher apresentaram o índice AGM muito próximo à média esperada para a população normativa, enquanto o resultado do AGC apresentou-se abaixo da média normativa com diferença de significância estatística $(p<0,001)$. Com relação a magnitude da diferença entre os homens agressores e os dados normativos, um tamanho de efeito médio foi observado para o AGC $(d=0,64)$ e um pequeno para o $\operatorname{AGM}(d=0,10)$, sugerindo quase nenhuma diferença entre os homens autores de violência contra a mulher e a amostra normativa para esse último código.

Tabela 3

Diferença de Média nos Indicadores de Agressividade no R-PAS em Amostras Distintas e Tamanho do Efeito ( $n=31$ )

\begin{tabular}{|c|c|c|c|c|}
\hline \multirow[t]{2}{*}{ Variáveis no R-PAS } & $\begin{array}{c}\text { Grupo } \\
\text { normativo }\end{array}$ & $\begin{array}{l}\text { Grupo de homens autores } \\
\text { de violência contra a mulher }\end{array}$ & \multirow[t]{2}{*}{$(g l) t$} & \multirow[t]{2}{*}{$d$} \\
\hline & $M(D P)$ & $M(D P)$ & & \\
\hline AGC & $3,1(1,9)$ & $1,9(1,7)$ & $(30)=-3,6^{* * *}$ & 0,64 \\
\hline AGM & $0,5(0,8)$ & $0,6(1,2)$ & $(30)=0,6$ & 0,10 \\
\hline
\end{tabular}

Notas. Nível de significância: ${ }^{* * *} p<0,001$

No caso do AGC, os valores apresentados pelo grupo de agressores foram mais baixos que os valores indicados pelo grupo normativo (homens autores de violência contra a mulher $M=1,9 ; D P=1,7$; normativo $M=3,1 ; D P=1,9 ; p=0,001, d$ de Cohen=0,64), permitindo inferir que os agressores apresentam baixo AGC, quando comparados à norma. Tais resultados não correspondem aos achados de Gacono et al. (2005) que apontam que o AGC é mais elevado em sujeitos com histórico de agressão, contudo ainda efetivo na discriminação entre grupos.
No que concerne o AGM, os resultados não permitem inferir que este seria um código que difere o grupo de agressores do grupo normativo, haja vista as médias muito próximas e um tamanho de efeito pequeno e não significativo (homens autores de violência contra a mulher $M=0,6 ; D P=1,2$; normativo $M=0,5 ; D P=0,8$; $p=0,52, d$ de Cohen $=0,10)$. Tais resultados, nessa amostra investigada, são fracos e, portanto, mostram-se limitados para identificar autores de violência, não sendo possível corroborar os achados de outros estudos com o SC (Benjestorf et al., 2013; Exner, 2003) que indicam que 
criminosos violentos, em contexto coercitivo, apresentam um menor número de respostas AG quando comparadas aos dados normativos. Contudo, os resultados desta investigação corroboram a interpretação proposta pelo manual do R-PAS que afirma que não existe evidência garantida de que o AGM possa estar relacionado à agressividade manifestada.

A diferença de resultados entre AGM e AGC, nessa amostra, também não parece explicada somente pelo fator coercitivo do contexto, na medida em que os agressores teriam evitado apenas conteúdos agressivos (baixo AGC comparado ao grupo normativo), mas não os movimentos agressivos (AGM). A explicação mais provável segue os achados da literatura que discutem a violência em relação ao fato de que gera incômodo ou sofrimento (caráter egodistônico do ato) ou como um comportamento banal a que estão acostumados em seus contextos de vida e que, portanto, não gera incômodo (caráter egossintônico) e passam despercebidos (Gacono, \& Meloy, 1994). Assim, infere-se, neste estudo, que a análise idiográfica do contexto de vida, com vivências de agressividade, do avaliado, pode explicar melhor os resultados, do que a simples interferência do ambiente coercitivo. Nessa perspectiva, é possível inferir que a amostra de agressores estudada apresentou respostas que indicavam menor tensão e preocupação com conteúdos agressivos, sugerindo processos de identificação de caráter egossintônico de seus comportamentos.

Para a compreensão desses dados de pesquisa, é necessário que se retome a própria natureza do fenômeno estudado. Discutir a violência exige um olhar mais amplo, que necessariamente inclua referências culturais e históricas do grupo social que define o que deve ser considerado como suportável ou não (Silva et al. 2015). Nesse sentido, o trabalho de intervenção com homens autores de violência contra a mulher tem dado ênfase na compreensão dos comportamentos violentos por meio de vivências passadas de violência e trauma que possam ter delimitado as relações interpessoais (Lima \& Büchele, 2011). Esses homens geralmente reconhecem apenas os excessos em sua agressividade e não avaliam sua agressão como um ato equivocado e exagerado de tentar corrigir o comportamento do outro (Lamoglia \& Minayo, 2009). O R-PAS não se propõe a avaliar a consciência do agressor em relação ao ato violento, mas uma pesquisa feita com homens autores de violência conjugal, vinculados a um Programa de Atenção à Violência Doméstica e Intrafamiliar, indicou que eles não percebem que a ação violenta contra a sua companheira os caracteriza como autores de violência (Rosa, Boing, Buchele, Oliveira, \& Coelho, 2008), o que pode explicar a hipótese do caráter egossintônico proposto. Esse tipo de disposição foi também observado no contato com os participantes do presente estudo, quando buscavam sempre justificar suas ações para as pesquisadoras como um comportamento óbvio e apenas de reação a comportamentos prévios de suas companheiras. Portanto, em situações de avaliação do comportamento agressivo, fica recomendado que o avaliador busque outras fontes de informação, como entrevistas ou instrumentos de autorrelato, para melhor embasar a compreensão final do sujeito.

Este estudo trouxe uma importante contribuição quanto à necessidade de ampliar a investigação dos diferentes tipos de comportamentos agressivos, extrapolando a discussão até então centrada no transtorno de personalidade antissocial (Gacono \& Meloy, 1994; Gacono et al., 2008). Contudo, apresentou como limitação a amostra pequena e selecionada em uma única Vara de Violência Doméstica. É possível que em amostras maiores, procedentes de diferentes varas, ou até mesmo composta por diferentes tipos de agressores, que não de violência contra a mulher, os resultados possam ser diferentes.

A falta de estudos comparativos com amostra em contextos coercitivos (forense não simulados) e não coercitivos (clínico) precisa ser investigada de forma a confirmar que a baixa incidência de AGC não tenha sido provocada de forma exclusiva pelo interesse em se mostrar como uma pessoa não violenta no contexto do judiciário. Mais estudos talvez possam justificar a continuidade do uso do AGM na codificação do R-PAS já que apontariam evidências de validade de critério. Além disso, com mais estudos empíricos, utilizando o R-PAS na administração e levantamento, será possível esclarecer as contradições na literatura, em relação ao AGC e AGM, dando direção mais clara quanto à validade de interpretação desses indicadores. De qualquer modo, a partir desse estudo, confirma-se a importância da inclusão do código AGC no R-PAS, baseado na proposta de Gacono e Meloy (1994), pois apresenta maior evidência de validade que o AGM, atualmente em uso pelo SC.

\section{Referências}

Benjestorf, S. T. V., Viglione, D., Lamb, J. D., \& Giromini, L. G. (2013). Suppression of agressive Rorschach responses among violent offenders and nonoffenders. Journal of Interpersonal Violence, 28(15), 2981-3003. doi: 10.1177/0886260513488688.

Cicchetti, D. V. (1994) Guidelines, criteria and rules of thumb for evaluating normed and standardized assessment instruments on psychology. Psychological Assessment, 6(4), 284-290. doi: 10.1037/1040-3590.6.4.284

Cohen, J. (1988). Statistical power analysis for the behavioral sciences ( $\left.2^{\mathrm{a}} \mathrm{ed}.\right)$. Hillsdale: Lawrence Erlbaum Associates.

Erard, R. E. (2012). Expert testimony using the Rorschach performance assessment system in psychological injury cases. Psychological Injury and Law, 5(2), 122-134. doi: 10.1007/s12207-012-9126-7 
Erard, R. E., \& Evans, F. B. (2017). Preface. Em R. E. Erard \& F. B. Evans, The Rorschach in multimethod forensic assessment. (pp. x-xix). New York: Routledge.

Erard, R. E., Meyer, G. J., \& Viglione, D. J. (2014). Setting the record straight: Comment on Gurley, Piechowski, Sheehan, and Gray (2014) on the Admissibility of the Rorschach Performance Assessment System (R-PAS) in Court. Psychological Injury and Law, 7(2), $165-177$. doi: 10.1007/s12207-014-9195-x

Exner, J. E. (2003). The Rorschach: a comprehensive system. Basic foundations and principles of interpretation. (4ed.) New Jersey: Wiley \& Sons.

Exner, J. E., \& Sendín, C. (1997). Some Issues in Rorschach Research. European Journal of Psychological Assessment, 13(3), 155-163. doi: $10.1007 / 978-1-4939-3151-4$

Gacono, C. B., \& Meloy, J. R. (1994). The Rorschach Assessment of Aggressive and Psychopathic Personalities. New Jersey: Lawrence Erlbaum Associates.

Gacono, C. B., Evans, F. B., \& Viglione, D. J. (2008). Essencial issues in the forensic use of the Rorschach. Em C. B. Gacono \& B. Evans, The handbook of forensic Rorschach assessment (pp. 3-20). New York: Routledge.

Gacono, C. B., Bannatyne-Gacono, L., Meloy, J. R., \& Baity, M. R. (2005). The Rorschach extended aggression scores. Rorschachiana 27(1), 164-190. doi: 10.1027/1192-5604.27.1.164.

Katko, N. J., Meyer, G. J., Mihura, J. L., \& Bombel, G. (2010). A principal components analysis of Rorschach aggression and hostility variables. Journal of Personality Assessment, 92(6), 594-598. doi: 10.1080/00223891.2010.513309.

Kivisalu, T. M., Lewey, J. H., Shaffer, T. W., \& Canfield, M. L. (2016). An investigation of interrater reliability for the Rorschach Performance Assessment System (R-PAS) in a nonpatient U.S. sample. Journal of Personality Assessment, 98(4), 382-390. doi: $10.1080 / 00223891.2017 .1325244$

Kivisto, A. J., \& Swan, S. (2013). Rorschach measures of aggression: A laboratory-based validity study. Journal of Personality Assessment, 95(1), 38-45. doi: 10.1080/00223891.2012.713882.

Lamoglia, C. V. A., \& Minayo, M. C. S. (2009). Violência conjugal, um problema social e de saúde pública: estudo em uma delegacia do interior do Estado do Rio de Janeiro. Ciência \& Saúde Coletiva, 14(2), 595-604. doi: 10.1590/S1413-81232009000200028.

Lima, D. C., \& Büchele, F. (2011). Revisão crítica sobre o atendimento a homens autores de violência doméstica e familiar contra as mulheres. Physis: Revista de Saúde Coletiva 2(21), 721-743. doi: 10.1590/S0103-73312011000200020.

Meyer, G. J., Viglione, D. J., Mihura, J. L., Erard, R. E., \& Erdberg, P. (2017). R-PAS - Sistema de Avaliação por Performance no Rorschach (1a.). São Paulo: Hogrefe.

Mihura, J. L., Meyer, G. J., Bombel, G., \& Dumitrascu, N. (2015). Standards, accuracy, and questions of bias in Rorschach Meta-Analyses: Reply to Wood, Garb, Nezworski, Lilienfeld, and Duke (2015). Psychological Bulletin, 141(1), 250-260. doi: 10.1037/a0038445

Mihura, J. L., Meyer, G. J., Dumitrascu, N., \& Bombel, G. (2013). The validity of individual Rorschach variables - Systematic reviews and meta-analyses of the Comprehensive System. Psychological Bulletin, 139(3), 548-605. doi: 10.1037/a0029406

Mihura, J. L., Meyer, G. J., Dumitrascu, N., \& Bombel, G. (2016). On conducting construct validity meta-analyses for the Rorschach: A reply to Tibon Czopp and Zeligman (2016). Journal of Personality Assessment, 98(4), 343-350. doi: 10.1080/00223891.2016.1158182

Nørbech, P. C. B., Fodstad, L., Kuisma, I., Lunde, K. B., \& Hartmann, E. (2016). Incarcerated violent offenders' ability to avoid revealing their potential for violence on the Rorschach and the MMPI-2. Journal of Personality Assessment, 98(4), 419-429. doi: 10.1080/00223891.2015.1129613

Pignolo, C., Giromini, L., Ando, A., Ghirardello, D., Girolamo, M. Di, Ales, F., \& Zennaro, A. (2016). An interrater reliability study of Rorschach Performance Assessment System (R-PAS) raw and complexity-adjusted scores. Journal of Personality Assessment, 99(3), 619625. doi: 10.1080/00223891.2017.1296844

Rosa, A. G., Boing, A. F., Buchele, F., Oliveira, W. F., \& Coelho, E. B. S. (2008). A violência conjugal contra a mulher a partir da ótica do homem autor da violência. Saúde e Sociedade, 17(3), 152-160. doi:10.1590/S0104-12902008000300015.

Rovinski, S. L. R. (2013). Fundamentos da perícia psicológica forense. (3 $3^{\mathrm{a}}$ ed.). São Paulo: Vetor.

Silva, A. C. L. G., Coelho, E. B. S., \& Moretti-Pires, R. O. (2014). O que se sabe sobre o autor de violência contra a parceira íntima: uma revisão sistemática. Revista Panamericana de Salud Publica, 35(4), 278-283. Recuperado de http://www.scielosp.org/pdf/rpsp/v35n4/07.pdf

Silva, F. A., Silva, F. P. P., Tavares, E. S., Oliveira, H. S. G., Neves, A. L. M., Silva, I. R., \& Oliveira, K. N. L. (2015). Atenção psicossocial a homens autores de violência conjugal contra a mulher: uma construção participativa. Pesquisas e Práticas Psicossociais, 10(1), $177-191$. Recuperado de https://goo.gl/mtEG6W

Society for Personality Assessment (2005). The status of the Rorschach in clinical and forensic practice: An official statement by the board of trustees of the Society for Personality Assessment. Journal of Personality Assessment, 85(2), 219-237. doi: 10.1207/s15327752jpa8502_16

Viglione, D. J., \& Meyer, G. J. (2008). An overview of Rorschach psychometrics for forensic practice. Em C. B. Gacono, F. B. Evans, N. KaserBoyd, \& L. A. Gacono (Eds.), The Handbook of Forensic Rorschach Assessment (pp. 21-53). New York: Routledge/Taylor \& Francis Group.

Weiner, I. B. (2001). El valor del Rorschach em la clínica y em la práctica forense. Trabalho apresentado no XI Congresso Latinoamericano de Rorschach y otras técnicas proyectivas. Rosário, Argentina. Recuperado de http://ochoa.freeservers.com/weiner1.htm

\section{Sobre os autores}

Sonia Liane Reichert Rovinski é psicóloga, Doutora em Psicologia pela Universidade de Santiago de Compostela (2003) e pósdoutora junto ao Grupo de Estudo, Aplicação e Pesquisa em Avaliação Psicológica (GEAPAP-UFRGS). Atua na área da psicologia forense, coordena o curso de Especialização em Psicologia Jurídica da Projecto - Centro Cultural e de Formação (RS) e o curso de Especialização em Psicologia Jurídica do Instituto Sapiens (PR).

Andréia Mello de Almeida Schneider é psicóloga e atualmente doutoranda no Programa de Pós-Graduação em Psicologia da Universidade Federal do Rio Grande do Sul (UFRGS), junto ao Grupo de Estudo, Aplicação e Pesquisa em Avaliação Psicológica (GEAPAPUFRGS). Atua na área clínica.

Juliane Pariz é psicóloga, especialista em Avaliação Psicológica (UFRS) e doutoranda em Psicologia (Griffith University, Austrália). Desenvolve pesquisas nas áreas de Avaliação da Personalidade no modelo dos Cinco Grandes Fatores e pela técnica de Rorschach; comportamento antissocial, agressivo e criminalidade na infância e adolescência.

Álvaro Zaneti Santos é psicólogo, atualmente realizando especialização em Terapia Sistêmica (InTCC) e em Neuropsicologia (InTCC/ Instituto de Neuropsicologia do Rio Grande do Sul).

Denise Ruschel Bandeira é psicóloga e doutora em Psicologia pela Universidade Federal do Rio Grande do Sul. Atualmente é professora e orientadora de mestrado e doutorado no Programa de Pós-Graduação em Psicologia da UFRGS, coordena o Grupo de Estudo, Aplicação e Pesquisa em Avaliação Psicológica (GEAPAP-UFRGS) e coordenadora do GT Avaliação Psicológica: Personalidade e Desenvolvimento Humano. 